\title{
Análisis de volatilidad y correlación entre Estados Unidos y Asia
}

\author{
Natàlia Valls Ruiz ${ }^{\mathrm{a}}$ \\ Departamento de Control de Riesgos de Mercado, \\ Caixa d'Estalvis i Pensions de Barcelona, Barcelona, España \\ natalia.valls@lacaixa.es \\ Helena Chuliá Soler ${ }^{b}$ \\ Departamento de Econometría, Estadística y Economía Española, \\ RFA-IREA, Universidad de Barcelona, Barcelona, España
}

\section{RESUMEN}

El presente artículo analiza la correlación entre Estados Unidos y Asia teniendo en cuenta el impacto de la crisis financiera actual. Dentro de los países asiáticos se escoge un mercado desarrollado, Japón, y distintos mercados emergentes, entre los cuales se encuentran los tigres asiáticos, los tigres menores y China. Los resultados empíricos muestran que a medida que el grado de desarrollo del mercado asiático analizado disminuye, la correlación con Estados Unidos es inferior.

Palabras clave: Correlación, Mercados financieros internacionales, crisis

Clasificación JEL: C58, G01, G15

a Departamento de Control de Riesgos de Mercado, Caixa d'Estalvis i Pensions de Barcelona, Barcelona, España. E-mail: natalia.valls@lacaixa.es

b Departamento de Econometría, Estadística y Economía Española, RFA-IREA, Universidad de Barcelona, Barcelona, España. E-mail: hchulia@ub.edu

Recibido: julio de 2010. Aceptado: septiembre de 2010 


\begin{abstract}
This paper analyses the behaviour of correlations between the US and the Asian stock markets taking into account the effect of the Global Financial crisis. Within the Asian markets, one Asian mature country, Japan, and ten emerging markets, which are the four Asian Tigers, the four Asian Tiger Cubs and China, are included in the sample. Empirical results show the level of correlations depends on the country's grade of development.
\end{abstract}

Keywords: Correlation, International financial markets, crises

JEL Classification: C58, G01, G15

\title{
1. INTRODUCCIÓN
}

En los últimos años, se ha podido observar cómo las economías emergentes han sido cada vez más sensibles a las noticias internacionales. Ante crisis financieras como la crisis asiática de 1997 o la crisis de la burbuja tecnológica a principio de este siglo, se ha constatado un incremento de la correlación de los mercados financieros de las economías emergentes con las economías desarrolladas. El presente artículo analiza si este patrón de aumento de la correlación se ha producido durante la última crisis financiera internacional.

Para ello, se toman los principales índices de acciones de distintos países, en concreto, entre un mercado maduro mundial, Estados Unidos, y diferentes mercados de Asia. Entre los países asiáticos, se encuentra un gran mercado desarrollado, como es Japón, y un segundo grupo formado por mercados emergentes. Dentro de este grupo de mercados emergentes consideramos por un lado, pertenecientes al sudeste asiático, los llamados tigres asiáticos o dragones de Asia y los tigres menores o pequeños dragones, y por otro lado China. A través del análisis de correlación que existe entre Estados Unidos y estos países, se podrá distinguir si el comportamiento es diferente según el grado de desarrollo de los países asiáticos. Asimismo, se analiza el impacto de la última crisis financiera en los mercados, todavía latente, y se comprueba si afecta de forma distinta en función del grado de desarrollo de los países estudiados.

Los países emergentes del sudeste asiático se dividen en dos grupos, los tigres asiáticos, en adelante tigres, y los tigres menores, en adelante menores. Los tigres 
surgieron entre 1945 y 1990, desarrollándose potencialmente Hong Kong (antigua colonia británica que actualmente pertenece a China), Corea del Sur, Singapur y Taiwán, países que sufrieron un gran crecimiento tanto en cantidad como en calidad de sus productos, logrando alcanzar en gran medida el mercado internacional. Los menores lograron su industrialización más tarde, integrados por Filipinas, Indonesia, Malasia y Tailandia. Tanto los tigres como los menores, son economías del sudeste asiático en crecimiento que presentan una gran oportunidad para la industria internacional, juntamente con China.

En la literatura financiera existen diversos estudios que analizan la transmisión de volatilidad entre mercados maduros y mercados emergentes (véase Aggarwal et al. (1999), Bessler y Yang (2003), Wong y Vlaar (2003), Syriopoulos (2004) entre otros). Algunos de ellos, como Beirne et al. (2009), Kuper y Lestano (2008), Ng (2000) y Mulyadi (2009), estudian las economías del sudeste asiático. Además de estudiar la transmisión de volatilidad entre mercados, diversos trabajos como los de Sheng y Tu (2000), Ratanapakorna y Sharma (2002), Jang y Sul (2002), Click y Plummer (2005), Caporale et al. (2006) y Lucey y Voronkova (2008) entre otros, también analizan el impacto de algunas crisis financieras en la transmisión de información entre mercados. La mayoría de los trabajos mencionados examinan el efecto de la crisis asiática de 1997.

No obstante, todavía no se han realizado estudios de correlación entre Estados Unidos y países emergentes del sudeste asiático teniendo en cuenta el efecto de la crisis financiera actual. En este sentido, este artículo se propone analizar si existe un antes y un después en el patrón de comportamiento de la correlación entre Estados Unidos y estos países. Para ello, se estima un modelo GARCH multivariante que permitirá obtener la correlación entre los diferentes países considerados y Estados Unidos y comprobar si el patrón de comportamiento es igual para cada país.

Los resultados muestran que existe un patrón general de comportamiento según el nivel de desarrollo económico del país analizado. Esto supone que el país más correlacionado con Estados Unidos es Japón, por su elevado grado de desarrollo. Dentro de los países emergentes del sudeste asiático, siguen los tigres y por detrás se encuentran los menores. Surgen algunas excepciones en este análisis, como son Filipinas, Corea del Sur y China.

El resto del artículo se estructura del siguiente modo. En la sección 2 se presentan y analizan los datos utilizados en el estudio. En la sección 3 se explica la metodología utilizada. En la sección 4 se presentan los resultados, tanto de volatilidades como de correlaciones. Finalmente, las conclusiones clausuran el trabajo. 


\section{DATOS}

Dentro de los países emergentes del sudeste asiático analizados, se distingue entre tigres y menores. Los tigres están compuestos por Corea del Sur (en adelante Corea), Taiwán, Hong-Kong y Singapur. Por otro lado, los menores, países que han mostrado un gran desarrollo en los últimos años y van justo por detrás de los tigres, son Malasia, Tailandia, Indonesia y Filipinas. Se incluye en este estudio China aunque no se encuentre dentro de los tigres porque el concepto de tigre obedece a país pequeño emergente con gran crecimiento. China no cumple la característica de país pequeño. Dado que se considera el motor de crecimiento de Asia en general, se ha decidido incluirlo en este análisis.

Los datos escogidos son los índices de acciones más representativos de los 11 mercados analizados, los cuales pueden agruparse de la siguiente forma: mercados maduros y mercados emergentes. Dentro de los maduros, se ha fijado el mercado maduro mundial como Estados Unidos, representado por su índice de mayor amplitud y representación, el S\&P500. Como mercado maduro asiático se ha escogido Japón, con el Nikkei 225 como índice más popular. En el segundo grupo se hallan los mercados emergentes, dentro de los cuales se encuentran los emergentes del sudeste asiático, con la distinción de dos grandes grupos: los tigres, con Hong-Kong (Hang Seng Index), Corea (Kospi Index), Singapur (Straits Time Index) y Taiwán (Taiwan Stock Exchange Index); los menores, con Filipinas (Philippine Stock Exchange Index), Indonesia (Jakarta Composite Index), Malasia (FTSE Bursa Malaysia Kuala Lumpur Composite Index) y Tailandia (FTSE SET Shariah Index). Finalmente, dentro de los mercados emergentes de Asia, se ha considerado interesante la inclusión China (Shanghai A-Share Stock Price Index). Cabe destacar que Hong-Kong, dentro del grupo de tigres, se distingue como mercado independiente de China por ser una reciente antigua colonia del Reino Unido, hasta el año 1997, manteniendo actualmente un sistema económico, administrativo y judicial independiente.

Por tanto, la muestra está formada por 11 mercados que serán analizados por parejas, con lo cual se formarán 10 parejas, incluyendo siempre Estados Unidos como mercado maduro a nivel mundial con el resto de mercados.

Los datos provienen de Bloomberg y el periodo muestral está comprendido entre el 1 de enero de 2003 y el 3 de marzo de 2010 (375 observaciones). De este modo se obvia la crisis de la burbuja tecnológica del año 2001 hasta 2002 y tan sólo se recoge el efecto de la crisis actual. Para ello, se introduce en el modelo una 
variable dicotómica dummy, que toma valor 1 a partir del día 15 de agosto de 2007 hasta la actualidad, y 0 el resto de días.

Varios analistas han fijado el inicio de la crisis financiera en agosto de 2007 porque fue cuando los bancos centrales de varios países tuvieron que intervenir para proporcionar liquidez en los mercados. A principios del mes de agosto de 2007 se anuncian algunas caídas de bancos importantes, tanto estadounidenses como europeos. La crisis de las hipotecas subprime está empezando a pisar fuerte en los mercados financieros. Los días 9 y 10 de agosto, esta crisis se traslada a todas las bolsas del mundo. Ante esta situación, los bancos centrales de Estados Unidos y de la Eurozona inyectan liquidez en el mercado interbancario para calmar la tensión producida por la gran falta de liquidez existente en el mercado financiero. Los siguientes días hasta el 15 de agosto, tanto la Reserva Federal de Estados Unidos, como el Banco Central Europeo, el Banco Central de Canadá y el Banco Central de Japón continúan inyectando liquidez. Por todo ello y dado que los datos recogen el cierre semanal de los miércoles, el día 15 de agosto de 2007 es el día que se considera en este estudio como inicio de la crisis en las bolsas de todo el mundo.

Se ha escogido utilizar cierres semanales para evitar el efecto del cambio horario entre países y se han calculado los rendimientos de miércoles a miércoles como diferencia de logaritmos para transformar las series en estacionarias:

$$
\text { rend. indice }=\ln \text { indice }_{t}-\ln \text { indice }_{t-1}=\ln \left(\frac{\text { indice }_{t}}{\text { indice }_{t-1}}\right)
$$

\subsection{Estadísticos descriptivos de las series}

En la Tabla 1 se muestran los estadísticos descriptivos de todas las series de rendimientos. En primer lugar, se observa que las medias de las series son muy próximas a 0 . En cambio, las desviaciones típicas son superiores, lo cual muestra que existen variaciones respecto a la media, es decir, presencia de volatilidad en todas las series. Por otro lado, el coeficiente de asimetría muestra que todos los mercados presentan asimetría negativa o a la izquierda, dado que este coeficiente es negativo, lo cual es una característica de las series bursátiles de acciones, ya que éstas son más volátiles en los movimientos bajistas del mercado que en los alcistas. Asimismo, todas ellas son leptocúrticas (coeficiente de curtosis superior a 3), es decir, tienen las colas más pesadas que las de una distribución Normal, lo cual es también característico de este tipo de series. En cuanto a la normalidad, se puede observar como todos los p-valor del 
estadístico Jarque-Bera son 0 , lo que supone rechazar la hipótesis nula de normalidad, propio de nuevo de las series de acciones.

TABLA 1.-Estadísticos descriptivos de las series

\begin{tabular}{|l|c|c|c|c|c|}
\hline Mercados & Media & $\begin{array}{c}\text { Desviación } \\
\text { estándar }\end{array}$ & Asimetría & Curtosis & Normalidad \\
\hline EEUU & 0.001 & 0.024 & -1.067 & 10.897 & $\begin{array}{c}1042.691 \\
(0.000)\end{array}$ \\
\hline Japón & 0.000 & 0.032 & -0.835 & 9.108 & $\begin{array}{c}624.805 \\
(0.000)\end{array}$ \\
\hline Hong-Kong & 0.002 & 0.035 & -0.348 & 6.572 & $\begin{array}{c}206.4242 \\
(0.000)\end{array}$ \\
\hline Corea & 0.003 & 0.035 & -0.278 & 7.871 & $\begin{array}{c}374.591 \\
(0.000)\end{array}$ \\
\hline Singapur & 0.002 & 0.030 & -0.157 & 8.075 & $\begin{array}{c}402.861 \\
(0.000)\end{array}$ \\
\hline Taiwan & 0.001 & 0.033 & -0.287 & 5.336 & $\begin{array}{c}90.177 \\
(0.000)\end{array}$ \\
\hline Filipinas & 0.003 & 0.033 & -0.434 & 5.188 & $\begin{array}{c}86.34322 \\
(0.000)\end{array}$ \\
\hline Indonesia & 0.005 & 0.039 & -1.165 & 10.859 & $\begin{array}{c}1047.005 \\
(0.000)\end{array}$ \\
\hline Malasia & 0.002 & 0.021 & -0.173 & 5.760 & $\begin{array}{c}120.569 \\
(0.000)\end{array}$ \\
\hline Tailandia & 0.002 & 0.034 & -0.643 & 8.619 & $\begin{array}{c}517.862 \\
(0.000)\end{array}$ \\
\hline China & 0.002 & 0.039 & -0.201 & 3.703 & $\begin{array}{c}10.215 \\
(0.006)\end{array}$ \\
\hline
\end{tabular}

Nota: Los valores entre paréntesis son los p-valor del estadístico Jarque-Bera.

En la Tabla 2 se pueden observar las correlaciones incondicionales de todas las series de rendimientos. Si se analizan las correlaciones de Estados Unidos con el resto de países, se observa que las más elevadas son las correlaciones con los países asiáticos más desarrollados. A medida que el nivel de desarrollo del país es menor, inferior es el grado de correlación con Estados Unidos. Entre los países asiáticos, se puede constatar un patrón general de correlación, asimismo en función del desarrollo económico de los países, según si se trata de Japón, de los tigres, de los menores o de China. 


\begin{tabular}{|c|c|c|c|c|c|c|c|c|c|c|c|}
\hline Mercados & EEUU & Japón & $\begin{array}{c}\text { Hong- } \\
\text { Kong }\end{array}$ & Corea & Singapur & Taiwan & Filipinas & Indonesia & Malasia & Tailandia & China \\
\hline EEUU & 1 & & & & & & & & & & \\
\hline Japón & 0.572 & 1 & & & & & & & & & \\
\hline $\begin{array}{l}\text { Hong- } \\
\text { Kong }\end{array}$ & 0.604 & 0.701 & 1 & & & & & & & & \\
\hline Corea & 0.438 & 0.702 & 0.706 & 1 & & & & & & & \\
\hline Singapur & 0.620 & 0.741 & 0.835 & 0.686 & 1 & & & & & & \\
\hline Taiwan & 0.461 & 0.598 & 0.660 & 0.658 & 0.642 & 1 & & & & & \\
\hline Filipinas & 0.377 & 0.519 & 0.525 & 0.519 & 0.581 & 0.491 & 1 & & & & \\
\hline Indonesia & 0.410 & 0.615 & 0.670 & 0.644 & 0.686 & 0.549 & 0.591 & 1 & & & \\
\hline Malasia & 0.399 & 0.562 & 0.637 & 0.563 & 0.682 & 0.539 & 0.591 & 0.640 & 1 & & \\
\hline Tailandia & 0.432 & 0.592 & 0.625 & 0.626 & 0.633 & 0.542 & 0.559 & 0.663 & 0.557 & 1 & \\
\hline China & 0.190 & 0.213 & 0.392 & 0.255 & 0.325 & 0.274 & 0.296 & 0.318 & 0.378 & 0.243 & 1 \\
\hline
\end{tabular}


La Tabla 3 muestra el contraste de raíz unitaria de Dickey-Fuller para todas las series en niveles. Se corrobora que todas las series son no estacionarias en media, dado que en todos los casos el p-valor es superior a 0.05 , es decir, que no se rechaza la $\mathrm{H}_{0}$ de que existe una raíz unitaria. Los valores críticos a los niveles de significación del $1 \%$, $5 \%$ y $10 \%$ son $-3.447627,-2.869050$ y -2.570838 , respectivamente. En todos los casos, el valor del estadístico es superior a estos tres valores.

Una vez obtenidas las series de rendimientos aplicando la diferencia de logaritmos mencionada previamente, se ha vuelto a realizar el test de Dickey-Fuller (Tabla 3) y se observa que todas las series ya son estacionarias, siendo todos los p-valor igual a 0 , rechazando así la hipótesis nula de no estacionariedad. Asimismo, el valor del estadístico es inferior a los valores críticos del $1 \%, 5 \%$ y $10 \%$.

TABLA 3.--Valor del estadístico de Dickey-Fuller (1981)

\begin{tabular}{|l|c|c|}
\cline { 2 - 3 } \multicolumn{1}{c|}{} & \multicolumn{2}{c|}{ Estadístico Dickey-Fuller } \\
\cline { 2 - 3 } & Serie en niveles & Serie de rendimientos \\
\hline \multirow{2}{*}{ EEUU } & -1.711936 & -19.36494 \\
& $(0.4244)$ & $(0.0000)$ \\
\hline \multirow{2}{*}{ Hopón } & -1.416932 & -18.97376 \\
& $(0.5744)$ & $(0.0000)$ \\
\hline \multirow{2}{*}{ Corea } & -1.560919 & -20.01315 \\
& $(0.5016)$ & $(0.0000)$ \\
\hline \multirow{2}{*}{ Singapur } & -1.447353 & -15.86237 \\
& $(0.5593)$ & $(0.0000)$ \\
\hline \multirow{2}{*}{ Taiwan } & -1.570158 & -19.57558 \\
& $(0.4969)$ & $(0.0000)$ \\
\hline \multirow{2}{*}{ Filipinas } & -1.954937 & -19.40887 \\
& $(0.3070)$ & $(0.0000)$ \\
\hline \multirow{2}{*}{ Indonesia } & -1.440076 & -19.82506 \\
& $(0.5629)$ & $(0.0000)$ \\
\hline \multirow{2}{*}{ Malasia } & -0.795737 & -9.434649 \\
& $(0.8188)$ & $(0.0000)$ \\
\hline \multirow{2}{*}{ Tailandia } & -1.426571 & -17.23884 \\
& $(0.5696)$ & $(0.0000)$ \\
\hline \multirow{2}{*}{ China } & -2.448210 & -19.33012 \\
& $(0.1293)$ & $(0.0000)$ \\
\hline
\end{tabular}

Nota: Los valores entre paréntesis corresponden al p-valor del estadístico, según el criterio de MacKinnon (1996). 


\section{Metodología}

Para estimar la correlación entre Estados Unidos y el resto de países considerados, se ha utilizado un modelo GARCH multivariante. Por tanto tenemos 10 modelos bivariantes que incluyen Estados Unidos con cada uno de los países asiáticos.

Concretamente se utiliza una versión asimétrica del modelo BEKK [Baba et al. (1989), Engle and Kroner (1995) y Kroner and Ng (1998)]. Se escoge el modelo asimétrico para poder captar el comportamiento asimétrico de la volatilidad, tanto en los propios mercados como en la transmisión de volatilidad que se capta a través de los efectos cruzados de los modelos multivariantes.

El modelo toma la siguiente forma:

$$
H_{t}=C^{\prime} C+B^{\prime} H_{t-1} B+A^{\prime} \varepsilon_{t-1} \varepsilon_{t-1}^{\prime} A+G^{\prime} \eta_{t-1} \eta_{t-1}^{\prime} G+V^{\prime} \varepsilon_{t-1} \varepsilon_{t-1}^{\prime} V D_{t}
$$

donde $\mathrm{C}, \mathrm{A}, \mathrm{B}, \mathrm{G}$ y $\mathrm{V}$ son matrices de parámetros a estimar, siendo $\mathrm{C}$ triangular superior y definida positiva, $\mathrm{H}_{\mathrm{t}}$ es la matriz de varianzas y covarianzas condicional en t y $D_{t}$ es la variable dummy que recoge el efecto de la crisis financiera, que toma valor 0 hasta el 15 de agosto de 2007 (sin incluir) y valor 1 en adelante.

Para estimar el modelo de la ecuación (2) se supone que las series de innovaciones siguen una distribución normal condicional y se aplica el método de quasi-máxima verosimilitud. Bollerslev y Wooldridge (1992) demuestran que los errores estándar calculados mediante el método de quasi-máxima verosimilitud son robustos aunque se viole el supuesto de normalidad.

La ecuación (2) permite analizar la varianza condicional de los propios mercados así como entre mercados, gracias a los efectos cruzados. Por ello, se puede llevar a cabo un estudio de la correlación entre los mercados analizados. Asimismo, se puede observar el efecto de la crisis financiera en el comportamiento de la volatilidad.

La estimación del modelo se ha llevado a cabo en 2 pasos. En primer lugar, se han estimado las ecuaciones univariantes para Estados Unidos y a continuación se imponen los resultados en 10 sistemas bivariantes que incluyen Estados Unidos con cada uno de los países asiáticos. Por tanto, se ha restringido que los coeficientes de Estados Unidos sean los mismos para todas las estimaciones. 


\section{Resultados}

\subsection{Comportamiento de volatilidades}

A continuación se muestran los gráficos de las volatilidades agrupados según la clasificación establecida en este artículo. En primer lugar, Estados Unidos con Japón, en segundo lugar, Estados Unidos con los tigres, seguido del mercado maduro mundial con los menores y finalizando con el mercado estadounidense con China.

El Gráfico 1 muestra la volatilidad del mercado de Estados Unidos (S\&P500) y Japón (NIKKEI 225). Se puede observar como las volatilidades de ambos índices, especialmente la del mercado japonés, empiezan a aumentar a partir de agosto de 2007, llegando a su punto álgido a finales del año 2008. La volatilidad del mercado nipón incrementa con fuerza concretamente en septiembre de 2008, cuando se produjo la caída de Lehman Brothers. La volatilidad del índice estadounidense también incrementa en esas fechas, no obstante, no tan fuertemente como la del índice japonés.

GráFICO 1.-Volatilidades de EEUU y Japón

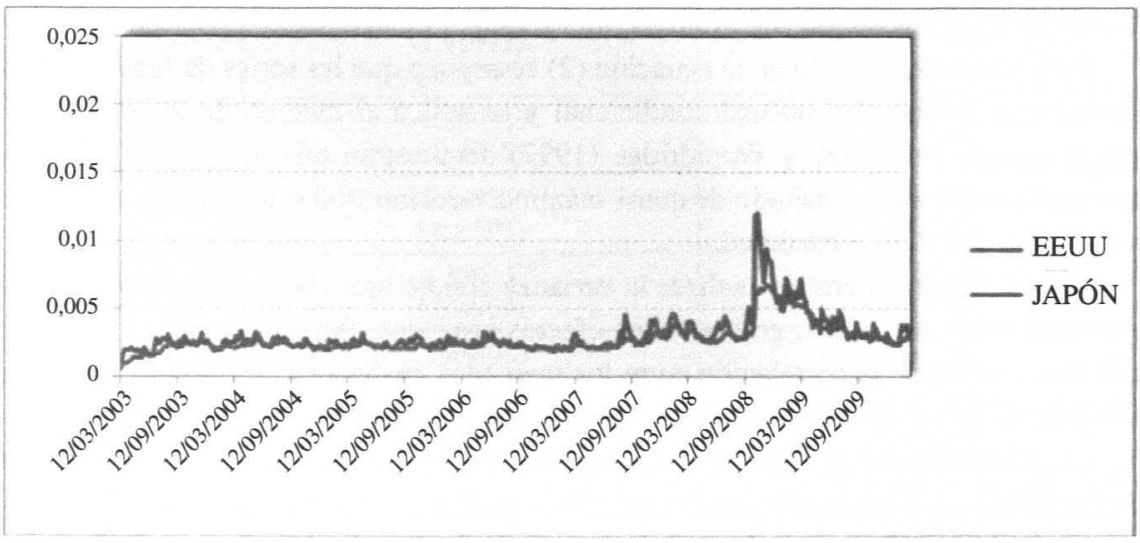

En el Gráfico 2, que muestra las volatilidades de Estados Unidos con respecto a los tigres, se observa de nuevo como todos los índices experimentan un incremento de volatilidad, que todavía no ha cedido a partir del inicio de la crisis en agosto de 2007. El patrón de comportamiento es el mismo que en el gráfico anterior, dado que las volatilidades de los cuatro índices de los tigres asiáticos experimentan un fuerte incremento, 
mucho más elevado del que experimenta el índice S\&P500, al producirse la quiebra del cuarto banco de inversión de Wall Street.

GrÁfICo 2.-Volatilidades de EEUU y los tigres

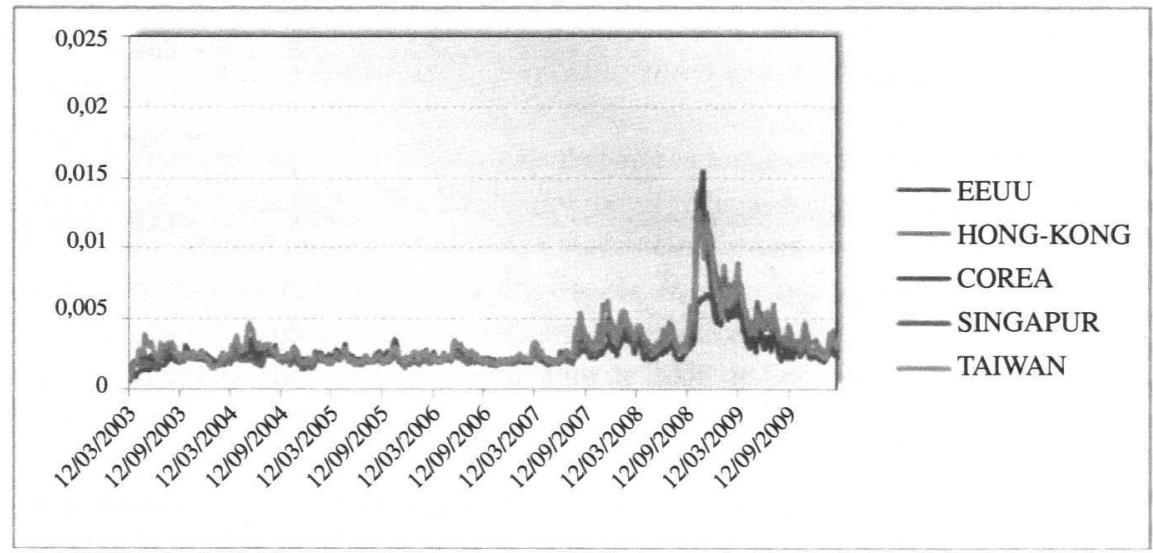

En cuanto a los menores, el Gráfico 3 muestra las volatilidades de éstos en comparación, de nuevo, con la del índice de mayor representación de Estados Unidos. En todos los mercados, la volatilidad empieza a incrementar a partir de agosto de 2007 y de nuevo alcanza su punto máximo a finales de 2008, consecuencia de la caída del banco Lehman Brothers. No obstante, se observa que Indonesia experimenta un fortísimo incremento de su volatilidad en esas fechas, del mismo modo que acusa mucho más que el resto de mercados el inicio de la crisis en el verano de 2007. En cambio, cabe destacar el comportamiento extremadamente similar que tiene Malasia en términos de volatilidad con respecto a Estados Unidos. 
GráFICo 3.--Volatilidades de EEUU y los menores

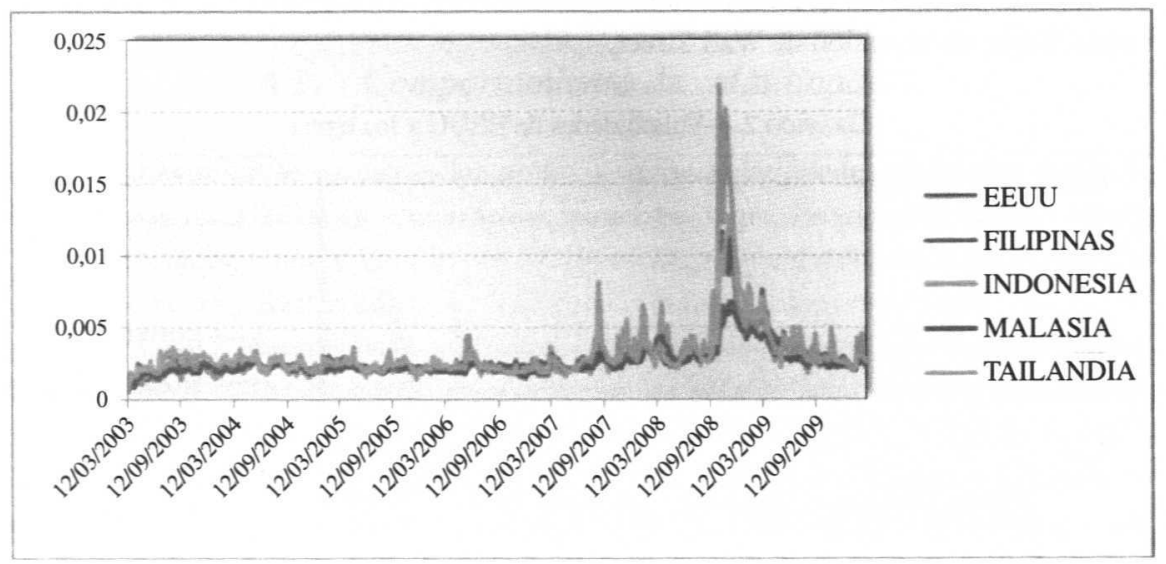

Finalmente, el comportamiento de la volatilidad de China en comparación con Estados Unidos (Gráfico 4), constata un comportamiento en general más similar al resto de mercados analizados, empezando con un aumento de volatilidad que todavía no ha cedido a partir del inicio de la crisis y llegando también a su cota máxima a finales de 2008, siempre por encima de la volatilidad de Estados Unidos.

GráfICo 4.--Vlatilidades de EEUU y China

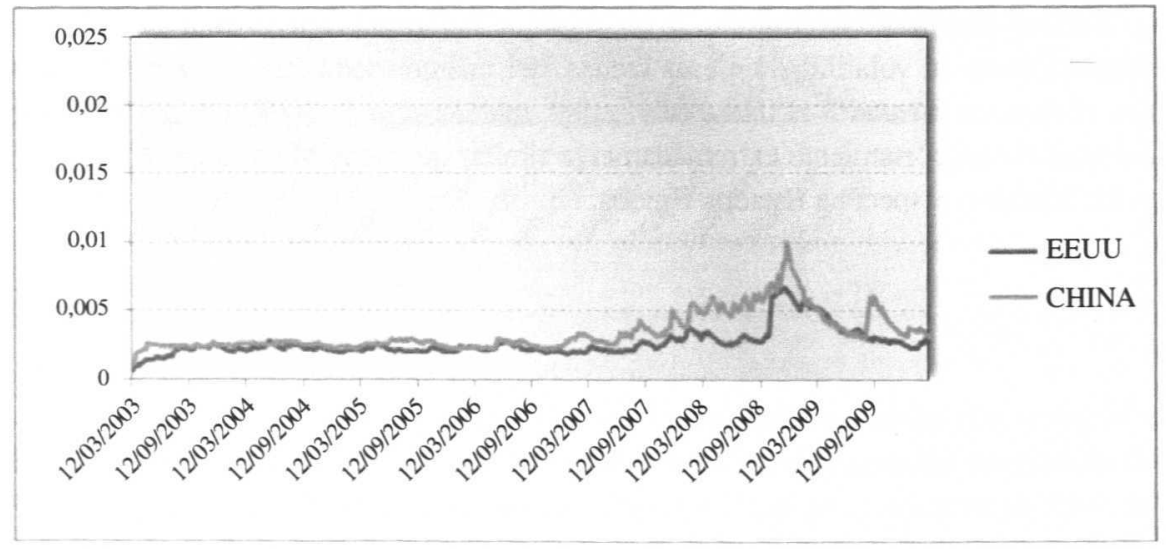


Cabe mencionar que se observa que la volatilidad de todos los mercados analizados es superior a la de Estados Unidos, característica típica de los mercados emergentes, que presentan elevados niveles de volatilidad.

\subsection{Análisis de correlaciones condicionales}

A continuación se muestra el análisis de correlaciones condicionales entre el principal índice de Estados Unidos y los principales índices del resto de mercados estudiados. El Gráfico 5 muestra el caso de Estados Unidos con Japón, cuyos índices han mantenido una correlación más o menos estable en el periodo analizado. Alrededor de los meses de septiembre y octubre de 2008 , momento en que la crisis llega a su punto más crítico con la caída, el 14 de septiembre de 2008 de Lehman Brothers, se observa que la correlación baja hasta el 0.76 , nivel que sigue siendo bastante elevado.

GRÁfICO 5.-Correlaciones condicionales de EEUU con Japón

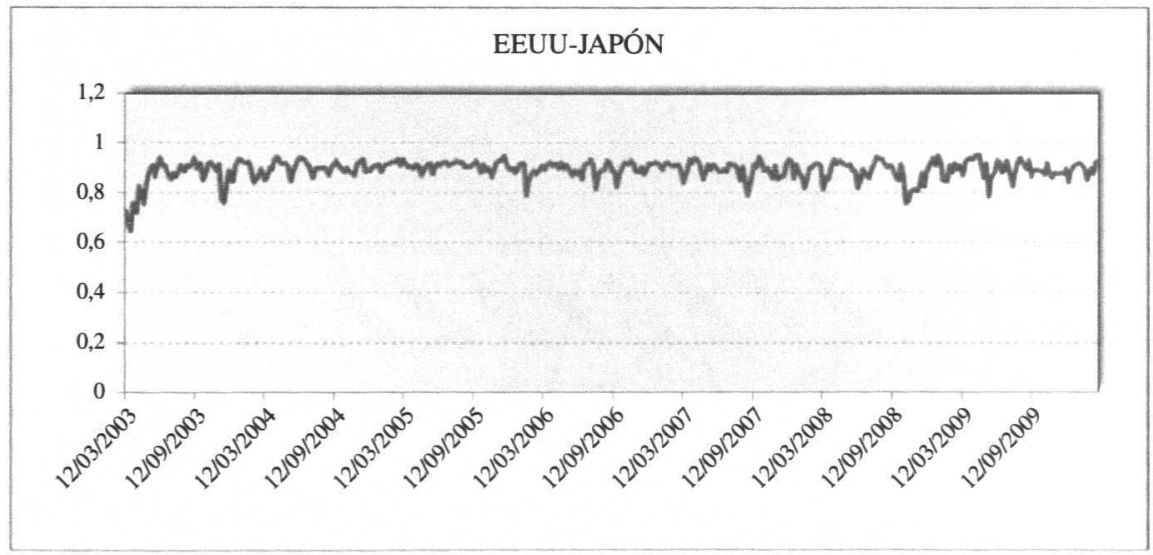

En el caso de Estados Unidos con Hong-Kong (Gráfico 6), la correlación en septiembre de 2008 cae hasta el 0.67 , es decir, un patrón similar al de Japón, pero la correlación disminuye un poco más. En el caso de Estados Unidos con Singapur y de Estados Unidos con Taiwán (Gráficos 9 y 10), ésta baja en esas fechas al 0.69 y al 0.71 , respectivamente. No obstante, es curioso el caso de Corea, en que la correlación con 
Estados Unidos disminuye bastante más (Gráfico 7), en concreto hasta el 0.39 el 5 de noviembre de 2008. Esto puede deberse al anuncio por parte del gobierno coreano el día 3 de noviembre de 2008 de la aplicación de un plan de estímulo de la economía por importe de 11 billones de dólares, destinados principalmente a rebajar la presión tributaria con el fin de impulsar el consumo de este país, por lo que los rendimientos del índice coreano Kospi mostraron un comportamiento discordante con el mercado estadounidense. En el Gráfico 8 se muestran los rendimientos de las semanas precedentes y posteriores a esta fecha, donde se puede comprobar que alrededor del 5 de noviembre de 2008 el comportamiento de Estados Unidos y Corea es completamente dispar. En general, se puede establecer un patrón de comportamiento similar para la correlación de los principales índices de acciones de los tigres con el S\&P500, excepto para Corea, probablemente por el motivo expuesto previamente.

GRÁFICo 6.-Correlaciones condicionales de EEUU con Hong-Kong

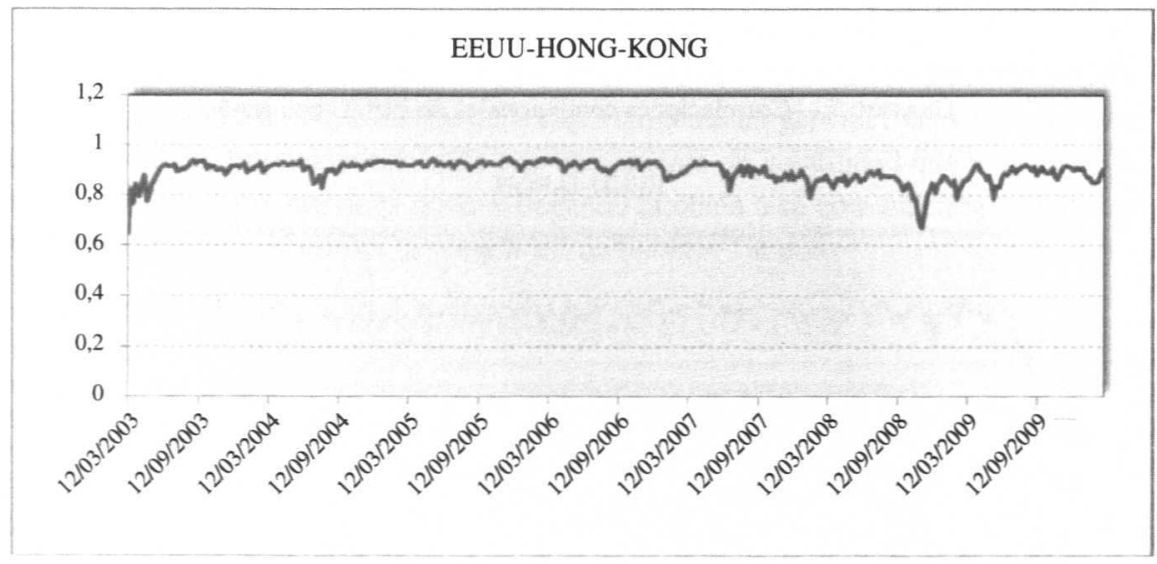


GRÁFICO 7.-Correlaciones condicionales de EEUU con Corea

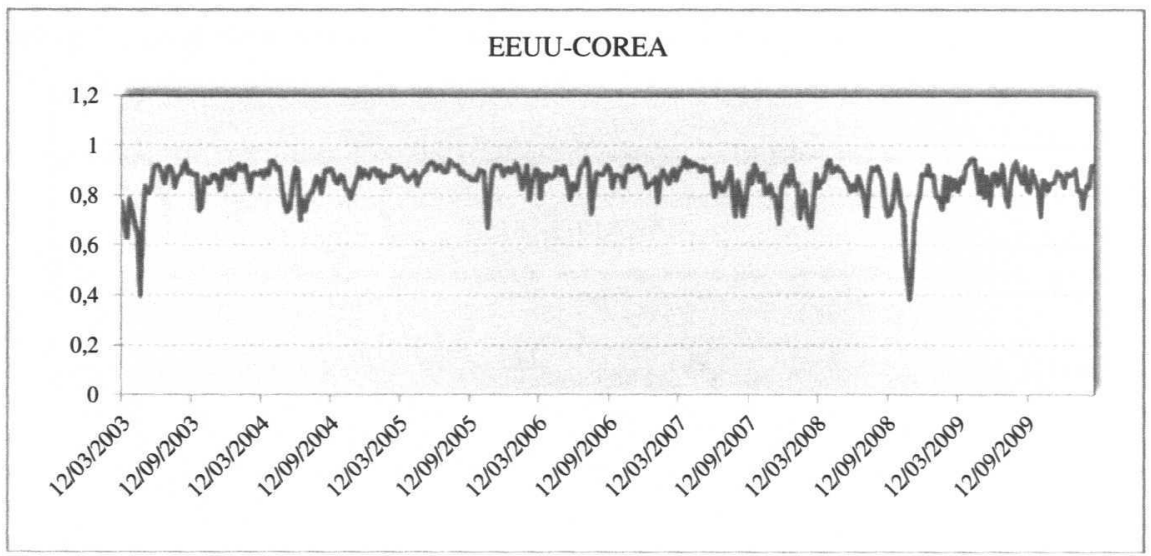

GRÁfICo 8.-Rendimientos de EEUU y Corea

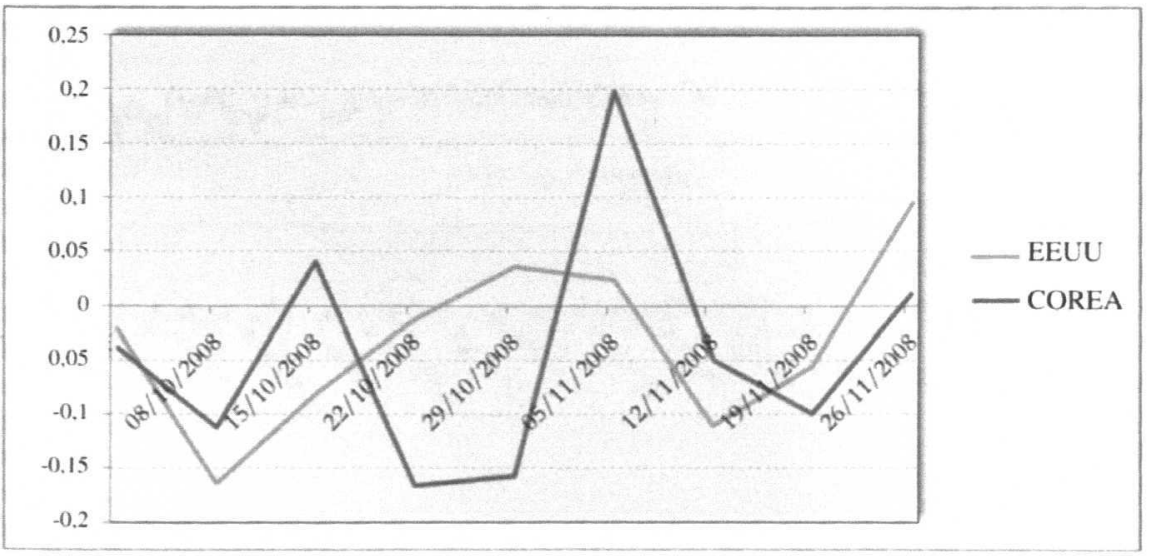


GRÁfICo 9.-Correlaciones condicionales de EEUU con Singapur

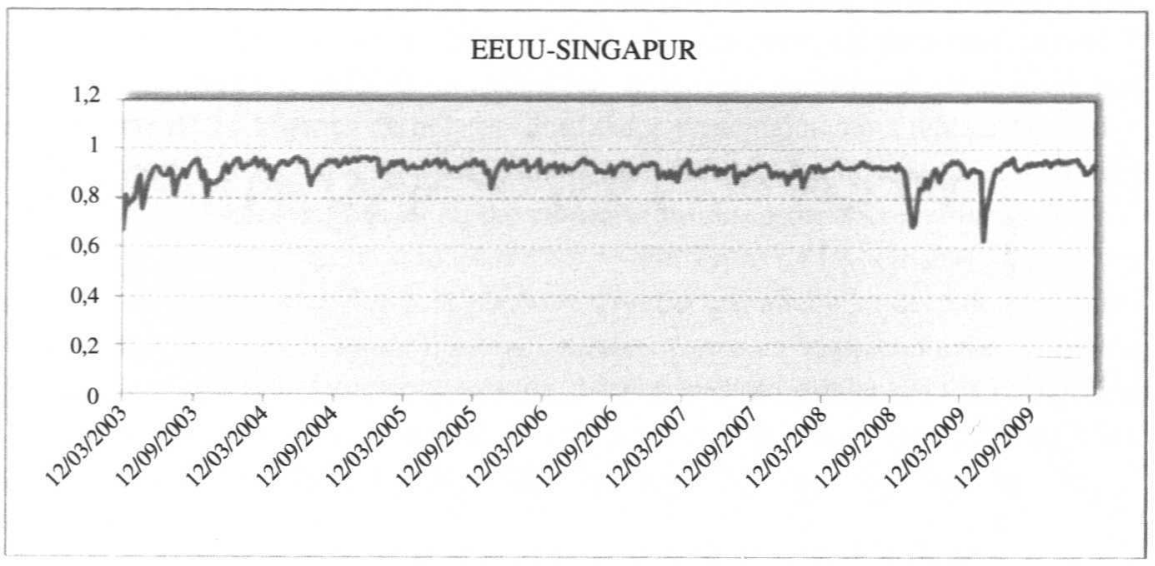

GRÁFICo 10.-Correlaciones condicionales de EEUU con Taiwán

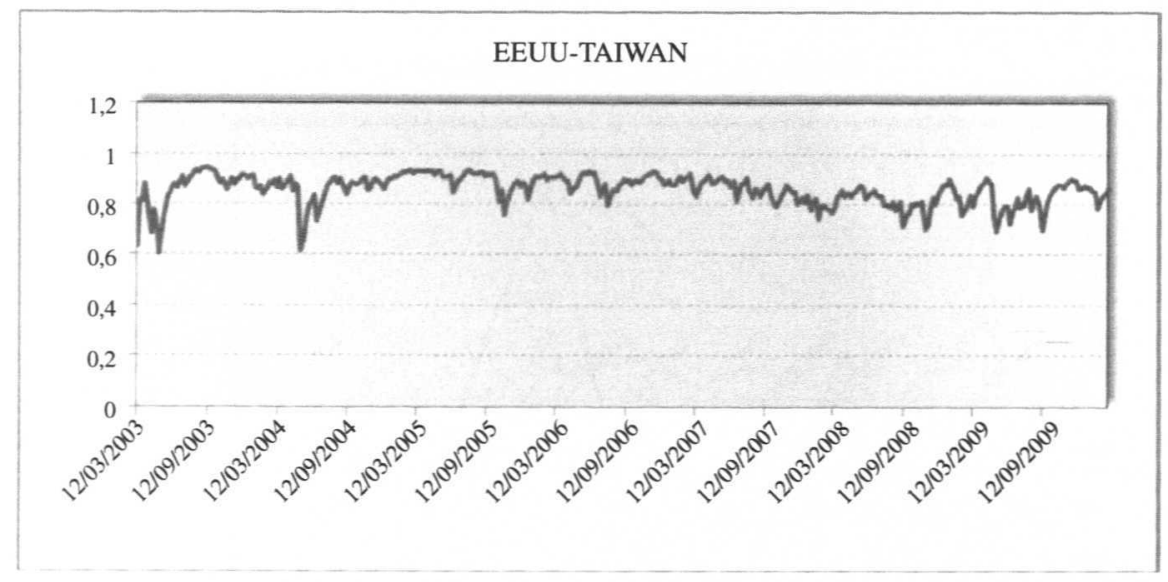

En cuanto a la correlación de los menores con Estados Unidos, se puede observar que ésta disminuye tras la caída del gran banco americano hasta el 0.66, 0.56, 0.61 y 0.56 para Filipinas, Indonesia, Malasia y Tailandia, respectivamente (Gráficos de 11 a 14). Los menores muestran un comportamiento similar entre ellos y sufren una caída en la correlación con Estados Unidos más fuerte que los tigres, por su menor grado de desarrollo. Cabe destacar que la correlación de Filipinas es la que menos disminuye por su elevada dependencia con 
Estados Unidos, dado que tiene una gran presencia de empresas estadounidenses por ser una antigua colonia americana (1901-1935), y por ello se comporta como un tigre.

GráfICo 11.-Correlaciones condicionales de EEUU con Filipinas

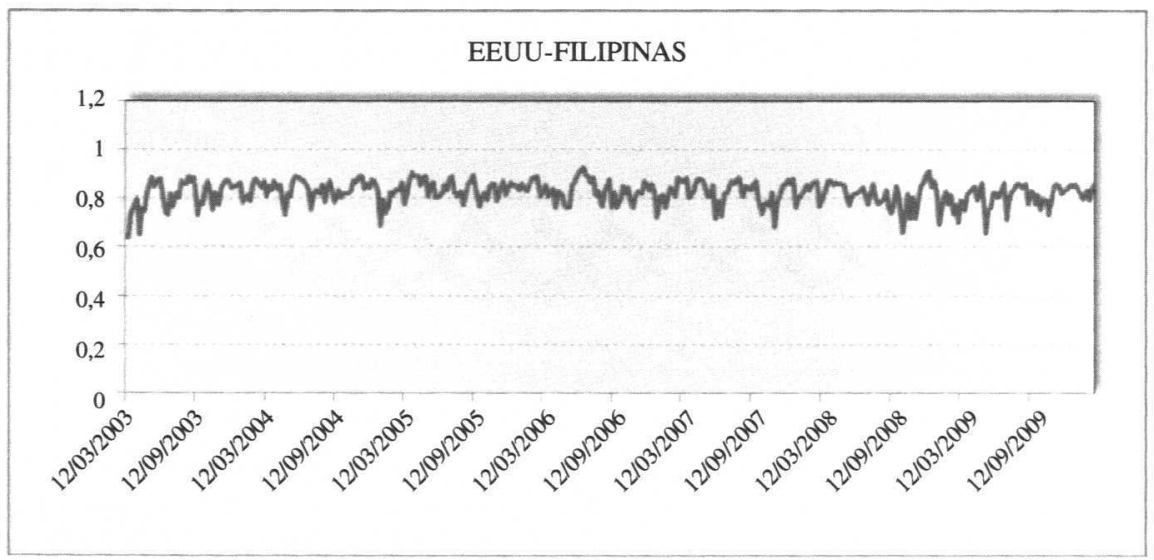

GRÁFICo 12.-Correlaciones condicionales de EEUU con Indonesia

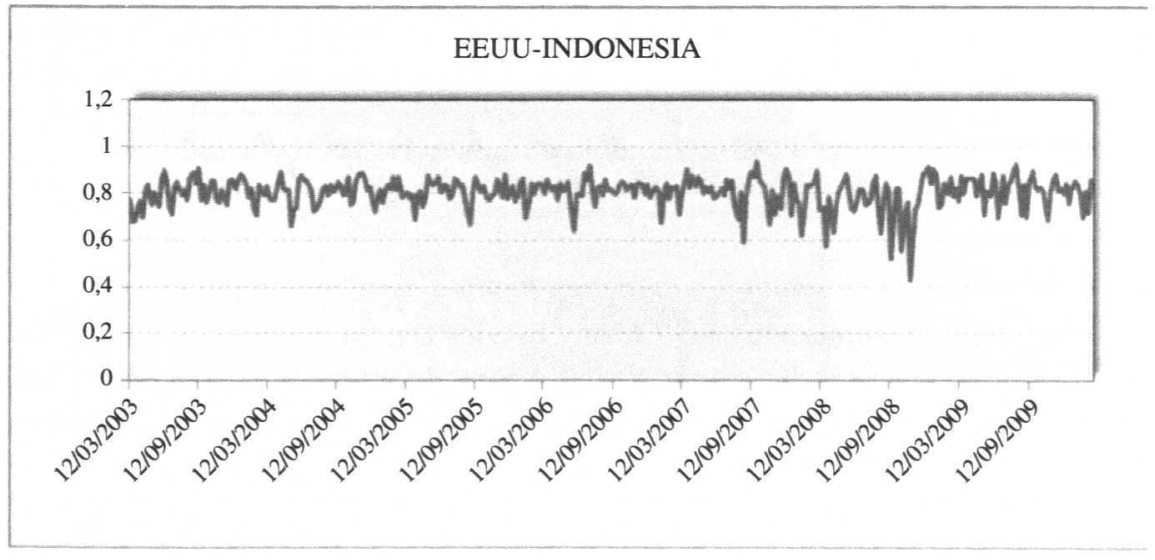


GRÁfICo 13.-Correlaciones condicionales de EEUU con Malasia

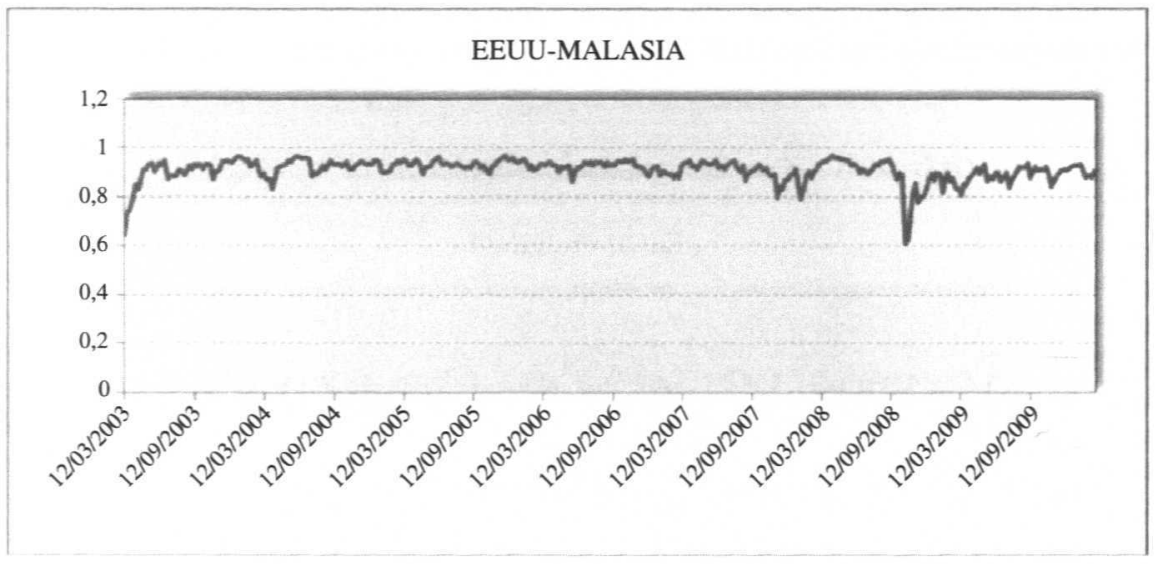

GRÁFICo 14.-Correlaciones condicionales de EEUU con Tailandia

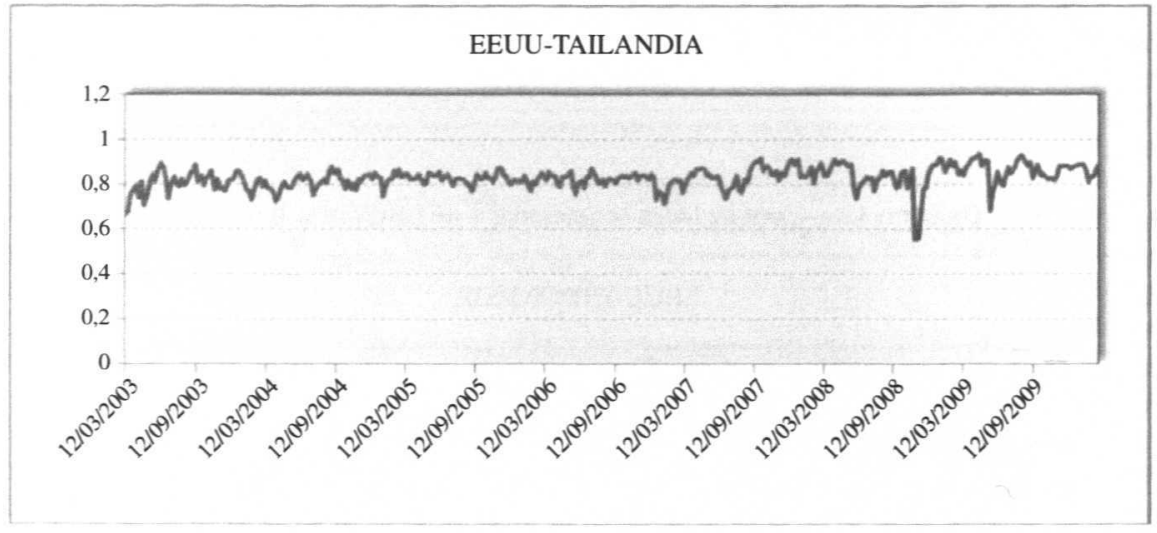

Finalmente, la correlación entre China y Estados Unidos (Gráfico 15) cae hasta el 0.53 , resultado que se podía esperar, ya que a pesar de ser un país muy grande y que crece a pasos agigantados, su sector financiero, como ya se ha comentado con anterioridad, es aún bastante cerrado. 
GRÁfICo 15.-Correlaciones condicionales de EEUU con China

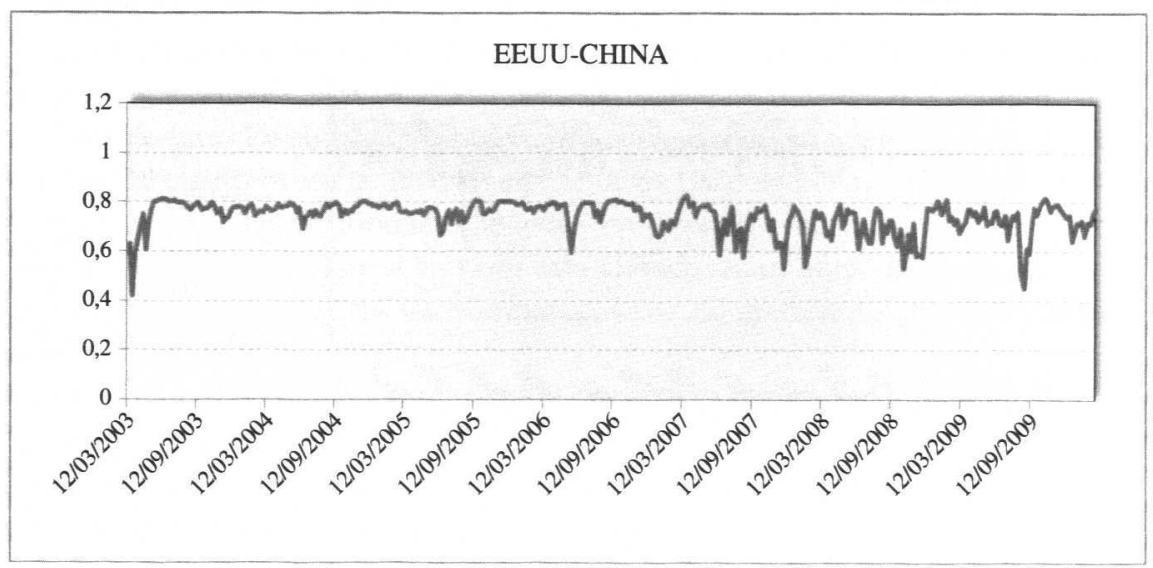

Se muestran ahora los gráficos de correlación condicional entre Estados Unidos y los países emergentes del sudeste asiático, agrupados en los dos grandes grupos de tigres y menores. En primer lugar, en el Gráfico 16, muestra la correlación de Estados Unidos con los tigres, observándose como la correlación cae de forma similar ante la quiebra de Lehman Brothers y un poco más que con Japón, excepto, como ya se ha comentado, para el caso de Corea.

En el caso de la correlación de Estados Unidos con los menores (Gráfico 17), ésta cae en esas fechas más que con los tigres, con un comportamiento similar entre ellos excepto para el caso de Filipinas, que como ya se ha explicado antes, se comporta como un tigre.

GRÁFICo 16.-Correlaciones condicionales de EEUU con los tigres

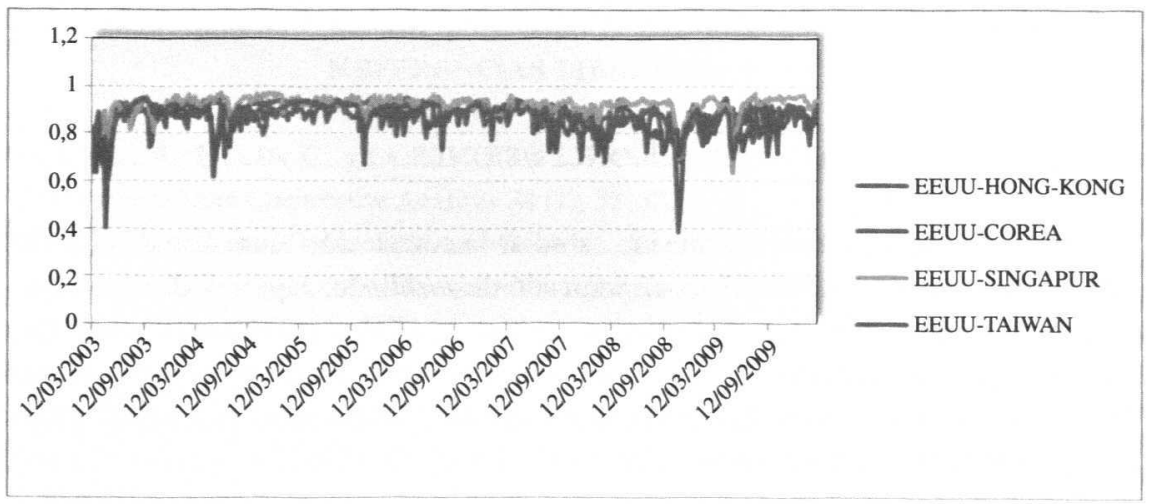


GRÁFICO 17.-Correlaciones condicionales de EEUU con los menores

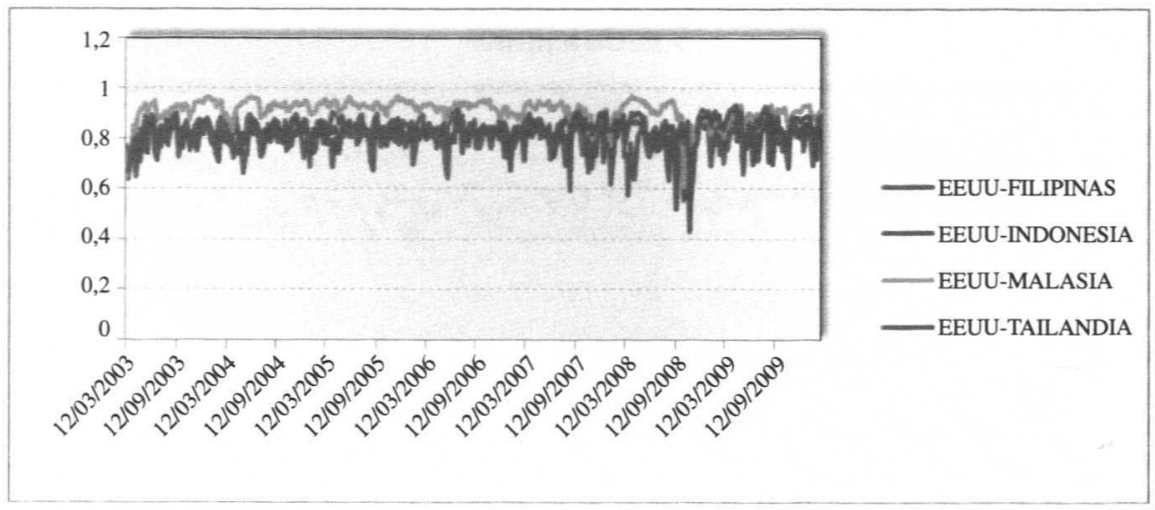

El análisis de las correlaciones condicionales de todos los mercados con el estadounidense muestra el siguiente patrón de comportamiento. Al producirse la quiebra del gran banco Lehman Brothers, la correlación de los mercados con el índice S\&P500 va disminuyendo cuanto menor es el grado de desarrollo de los países, es decir, que la correlación baja más en los países más emergentes y se mantiene o disminuye menos en los países con un grado de desarrollo mayor. Este resultado podría parecer contradictorio dada la mayor integración de la economía internacional de las últimas dos décadas. Sin embargo, el detonante de la crisis financiera actual no afectó a los países emergentes, puesto que los mercados con sistemas financieros menos desarrollados son ajenos a procesos de titulización en base a crédito y a su negociación. De este modo, se aislaron de la pérdida de valor que experimentaron estos activos a raíz de la crisis de las hipotecas subprime.

\section{Conclusiones}

El objetivo de este artículo ha sido estudiar la correlación entre Estados Unidos y Asia, analizando dentro de Asia un mercado desarrollado, Japón, y distintos mercados emergentes, entre los cuales se encuentran los tigres asiáticos, formados por Hong-Kong, Corea del Sur, Singapur y Taiwán, y los tigres menores, compuestos por Filipinas, Indonesia, Malasia y Tailandia. Finalmente, se completa el grupo de países emergentes asiáticos con China. 
Para llevar a cabo este análisis, se ha procedido a estimar un modelo GARCH multivariante asimétrico para la volatilidad. La estimación del modelo BEKK se ha hecho por parejas, siendo Estados Unidos el mercado que se ha estimado con el resto.

Cabe destacar los siguientes resultados. Se comprueba en todos los casos que, tras la caída del cuarto banco de inversión de Estados Unidos, Lehman Brothers, en septiembre de 2008, las volatilidades de todos los mercados incrementaron con fuerza, llegando en todos los casos a su nivel más elevado. Asimismo, las volatilidades de los mercados emergentes son las más elevadas en todo momento, característica típica de estas economías.

En cuanto a las correlaciones condicionales, se ha corroborado que a medida que el grado de desarrollo del mercado analizado disminuye, la correlación con Estados Unidos es inferior. Japón, como mercado desarrollado, tiene una elevada correlación con Estados Unidos, los tigres, con menor crecimiento presentan menor correlación y los menores, con un nivel de desarrollo aún inferior, muestran un menor grado de correlación con el mercado estadounidense. Sin embargo, destaca la baja correlación de China con Estados Unidos, debido al cerrado sector financiero que posee este gran país asiático.

Finalmente, cabe destacar que, a pesar de la mayor integración de las economías alcanzada en los últimos años, la crisis financiera actual ha afectado en menor medida a los mercados financieros de los países emergentes del sudeste asiático. El motivo obedece a que estos mercados poseen sistemas financieros menos sofistica dos, con lo cual, se han mostrado ajenos a la pérdida de valor sufrida por los activos de titulización de crédito que provocaron la propagación de la crisis desde Estados Unidos hacia los demás sistemas financieros desarrollados.

\section{REFERENCIAS BIBLIOGRÁFICAS}

AgGaRWAL, R., InCLAN, C., y LeAL, R. (1999). "Volatility in emerging stock markets". Journal of Financial and Quantitative Analysis 34 (1), 33-55.

Beirne, J., Caporale, G. M., Schulze-Ghattas, M. y Spagnolo, N. (2009): "Volatility Spillovers and Contagion from Mature to Emerging Stock Markets”. European Central Bank. Working Paper Series No. 1113.

Bessler, D. A. y YANG, J. (2003) : "The structure of interdependence in international stock markets". Journal of International Money and Finance 22 (2), 261-287.

Burns, P. y ENGLE, R. (1998): "Correlations and Volatilities of Asynchronous Data". Journal of Derivatives 5 (4), 7-18. 
Caporale, G. M., Pittis, N. y Spagnolo, N. (2006): "Volatility transmission and financial crisis“. Journal of Economics and Finance 30 (3), 376-390.

Click, R.W. y Plummer, M.G. (2005): "Stock market integration in ASEAN after the Asian financial crisis". Journal of Asian Economics 16 (1), 5-28.

Dickey, D. A. y W. A. Fuller (1981): "Likelihood Ratios Statistics for Autorregresive Time Series with a Unit Root”. Econometrica 49 (4), 1057-72.

ENGLE, R. F. y K. F. KRONER (1995): "Multivariate Simultaneous Generalized Arch”. Econometric Theory 11 (1), 122-150.

ENGLE, R. F. (1982): "Autoregressive Conditional Heteroskedasticity with Estimates of the Variance of UK Inflation”. Econometrica 50 (4), 987-1008.

Glosten, L. R., R. Jagannathan y D. E. Runkel (1993): "On the Relation between the Expected Value and Volatility of Nominal Excess Return on Stocks". Journal of Finance 48 (5), 1779-1801.

JANG, H. y Sul, W. (2002): "The Asian financial crisis and the co-movement of Asian stock markets". Journal of Asian Economics 13 (1), 94-104.

Kroner, K. F. y V. K. Ng (1998): "Modelling Asymmetric Comovements of Asset Returns". The Review of Financial Studies 11 (4), 817-844.

Lo, A. y A. C. MacKInlay, (1990): "An Econometric Analysis of Nonsynchronous Trading". Journal of Econometrics 45 (1-2), 181-211.

LuCEY, B. M. y Voronkova, S. (2008): "Russian equity market linkages before and after the 1998 crisis: evidence from stochastic and regime-switching cointegration tests". Journal of International Money and Finance 27 (8), 1303-1324.

MacKinnoN, J. G. (1996); "Numerical distribution functions for unit root and cointegration tests". Journal of Applied Econometrics 11, 601-618.

MUlYADI, M. S. (2009): "Volatility spillover in Indonesia, USA and Japan capital market". Munich Personal RePEc Archive. Paper No 16914.

NG, A. (2000): "Volatility spillover effects from Japan and the US to the Pacific-Basin". Journal of International Money and Finance 19 (2), 207-233.

RATANAPAKorn, O. y Sharma, S. (2002): "Interrelationships among regional stock indices". Review of Financial Economics 11 (2), 91-108.

SHENG, H. C. y TU, A. H. (2000): "A study of cointegration and variance decomposition among national equity indices before and during the period of the Asian financial crisis". Journal of Multinational Financial Management 10 (3-4), 345-365.

SYRIOPOULOS, T. (2004): "International portfolio diversification to Central European stockmarkets". Applied Financial Economics 14 (17), 1-16.

WONG, A. y VlaAR, P. (2003): "Modelling time-varying correlations of financial markets". Research Memorandum No. 739. De Nederlandsche Bank. 